\title{
Performance enhancement of Dicode Pulse Position Modulation System by means of LDPC code
}

\author{
Yasmeen M. Hussein ${ }^{1}$, Basman M. Al-Nedawe², Ammar Hussein Mutlag ${ }^{3}$ \\ \{bbc0030@mtu.edu.iq ${ }^{1}$, b.al-nedawe@mtu.edu.iq ${ }^{2}$, ammar_alqiesy@yahoo.com $\left.{ }^{3}\right\}$ \\ Middle Technical University, Electrical Engineering Technical College, Baghdad, Iraq 1,2,3 \\ Middle Technical University, Technical Institute of Baquba, Diyala, Iraq²
}

\begin{abstract}
Several types of Pulse Position Modulation (PPM) formats were proposed as coding schemes for optical communication such as Differential Pulse Position Modulation (DPPM) and Multiple Pulse Position Modulation (MPPM). However, these coding schemes result in a large bandwidth expansion factor that deduces an excessively high final data rate. On the other hand, Dicode Pulse Position Modulation (DiPPM) is an unconventional coding scheme that has superior characteristics beyond the PPMs proposed. More specifically, DiPPM has been developed to resolve the bandwidth consumption issue of PPM formats. For the first time, this study intends to overcome the DiPPM errors issue via the implication of forwarding Error Correction (FEC). In this regard, low-density parity-check (LDPC) is used in conjunction with multi-level coding, multi-stage encoding, and trellis-based signal shaping to create a bandwidth-efficient modulation format. The optimum code rates for different fibre normalised bandwidths of maximum transmission efficacy will be investigated.
\end{abstract}

Keywords: Dicode pulse position modulation (DiPPM); forward error correction (FEC) code; Low-density parity-check (LDPC); log-likelihood ratio (LLR); Bandwidth consumption issue.

\section{Introduction}

The communication device transmits data from the source to the receiver by a channel or transmitted media such as wired or wireless. The change and specifically the external noise of the channel are the main parameters controlling the reliability of obtained data. The noise is the main reason for signal interference and errors in transmitted data [1]. Channel coding is a smart way to increase interface performance in low-turbulence situations. In this regard, various types of PPM formats were presented in the literature as coding schemes for optical communication. For instance, MPPM and DPPM [2]. PPM is widely used in deep-space optical communication due to its high energy consumption, good anti-interference efficiency, and high detection frequency. However, one of the most drawbacks of PPMs is the production of an extremely high final data rate due to a large bandwidth expansion factor [3]. In other words, the final line rate may be extremely high, restricting its usefulness. Therefore, these coding schemes are appropriately used to synthesize directed lines of sight networks of glass optical fibre cables where bandwidth is not essential. In this aspect, optical fibre connections are widely used in low-bandwidth magnetic recording networks due to their inexpensive characteristics [4]. Thus, to resolve the challenge of bandwidth, DiPPM has been developed by Sibley (2003a) as an unconventional coding scheme that has superior characteristics beyond the PPMs proposed. More specifically, the DiPPM was developed to eliminate the main concern of bandwidth dissipation via the PPM.

LDPC codes are extensively used as efficient codes compared to other adaptable codes due to their applications with competent and reliable information transfer over bandwidth links in the existence of corrupting noise. Therefore, LDPC codes can operate in a wide range of channels. However, the reliability and accuracy of these codes and techniques deteriorate as the block length gets smaller. Only data transformations are sent in this signalling format, and no signal is sent with fixed data [5]. 
LDPC and PPM iterative demodulation systems are popular in deep space algorithms due to their output is close to the Shannon limit. The Shannon limit of a communication channel denotes the extreme rate of error-free data which can be transferred over the channel if the link is subjected to random data transmission errors, for a specific noise level. Reed Solomon code (RS) was also invented by Reed and Gustave Solomon in 1960 (Reed and Solomon, 1960) to control DiPPM error sources. Reed and Solomon (1960) confirmed the superiority of DiPPM to efficiently check the errors despite the packet error rate performance can be enhanced using the RS algorithm at the time of error detection. Additionally, the DiPPM errors acquired during the transmission can be eliminated using the MLSD error corrector [6].

Up to the authors' knowledge, the examination of DiPPM and LDPC code decoding techniques have not been yet applied to mitigate the issues of bandwidth expansion in PPM. Thus, this paper intends to resolve this challenge via incorporating the DiPPM and LDPC code to eliminate the errors and investigating the optimum parameters of LDPC.

\section{Dicode Pulse Position Modulation (DiPPM)}

To address the issue of bandwidth expansion in PPM, DiPPM was suggested [7]. DiPPMs have a similar trend to the PPMs formats. DiPPM has four-time slots, which was employed in the pulse code modulation (PCM) of a single time interval amplification. PCM approach helps to digitally signify sampled analogy signals. Specifically, the amplitude of the analogy signal is tested frequently at identical intervals for a PCM stream, and each sample is estimated to the nearest value within a range of digital steps.

A SET (S) pulse is sent to the 1st slot, while a SET (S) pulse is sent to the 2nd slot. The 2nd slot is used for a RESET (R) pulse, and the 3rd and 4th fourth slots are used as guard slots to prevent inter-symbol interference (ISI). Due to a PCM data inversion from 0 to 1 , the $S$ pulse is coded, whereas the $\mathrm{R}$ pulse is produced at a one-to-zero inversion. This is schematically presented in Fig. 1. Fig. 1 demonstrates the translation of PCM data from the top trace into a middle trace of dicode and bottom trace of DiPPM. The alphabet of DiPPM symbols is depicted in Table 1.

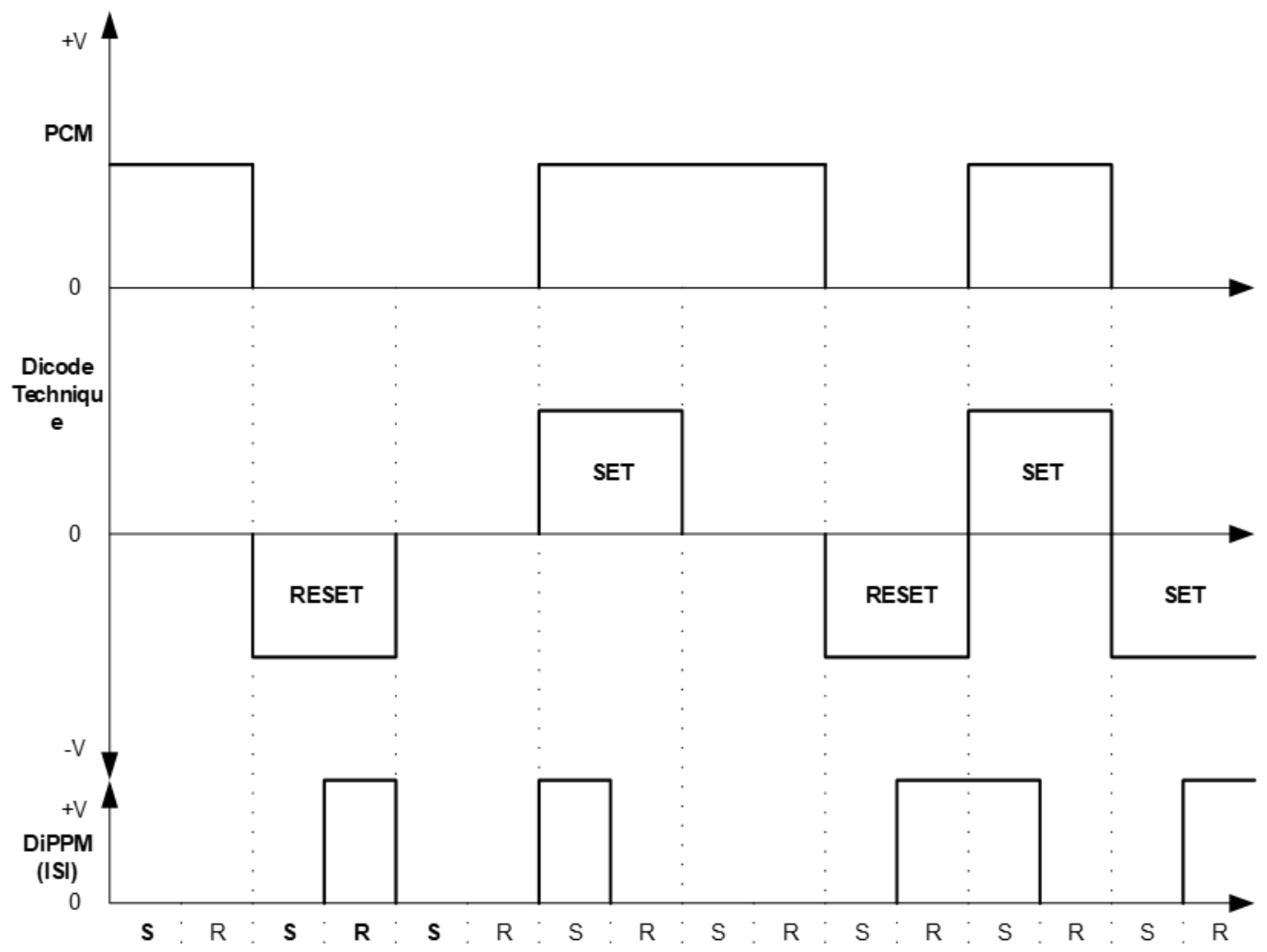

Fig 1. Translation of top trace (PCM data) into middle trace (Dicode) and bottom trace (DiPPM). 
Table 1. DiPPM symbol alphabet

\begin{tabular}{lll}
\hline $\mathrm{P}$ & DiPPM & $\mathrm{S}$ \\
$\mathrm{C}$ & & $\mathrm{y}$ \\
$\mathrm{M}$ & & $\mathrm{m}$ \\
& & $\mathrm{b}$ \\
& & $\mathrm{o}$ \\
\hline 0 & & 1 \\
0 & No & $\mathrm{N}$ \\
0 & pulse & \\
1 & SET & $\mathrm{S}$ \\
1 & & \\
0 & RESET & $\mathrm{R}$ \\
1 & NO & $\mathrm{N}$ \\
1 & Pulse & \\
\hline
\end{tabular}

Although the PCM data remains unchanged, no pulse is transmitted. However, the inter-symbol interference was minimised due to the implication of central decision detection to substitute the slope detection technique. In other words, the central decision detection technique has reduced the difficulty of the DiPPM decoder via deploying a simple 1st order preamplifier in cascade and a 3rd order Butterworth filter [8]. More specifically, the DiPPM's line rate was reduced to twice the real data because of the lack of guard bits. Thus, it is fair to confirm the viability of the DiPPM format to compete with other PPM formats implemented in optical fibre systems because of its high efficacy and simplicity.

\section{DiPPM ERROR}

Three types of detection errors can be found in DiPPM format including wrong-slot, erasure, and false alarm [9]. The next sections highlight the description and probabilities of these errors in detail.

\subsection{Wrong-Slot Errors}

Wrong-slot errors occur when noise on the rising edge of a detected pulse causes the pulse to be presented in contiguous time slots. Therefore, the pulse must be identified to reduce these errors in the middle of the Ts-width time slot. Also, a new pattern is formed when the edge transfers by [Ts/2].

\subsection{Erasure Errors}

Erasure errors of a pulse occur when the peak signal voltage falls under the threshold level. Specifically, this happens at a high level of noise.

\subsection{False-Alarm Errors}

False-alarm errors occur if the noise crosses a threshold limit in any unused data slot.

\section{Low-Density Parity Check (LDPC) Code}

The Low-Density Parity Check (LDPC) code has been proposed with a matrix of the parity search that has $\mathrm{N}$ columns and $\mathrm{M}$ rows and is represented as $\mathrm{H}=[\mathrm{HMxN}]$. LDPC code contains several 0 s and a restricted amount of the 1s. In other words, systematic LDPC codes have been deliberated with special characteristics. The characteristics specify that each column has a fixed "1" number such as weight of the column and each row has a fixed "1" number (i.e. weight of the row). However, there is no consistency between the weight of the column and the line weight. The LDPC code has been specified by the code of the LDPC (N,y, k). N signifies the recurrent, use a different code-word term length, and $\mathrm{j}, \mathrm{k}$ is the LDPC code's column and row 
weight, respectively. 1-y / $\mathrm{k}=1-\mathrm{M} / \mathrm{N}$ is the LDPC code rate. The 8-bit code length normal parity-check matrix is identified as [10]

$$
H=\left[\begin{array}{llllllll}
0 & 1 & 0 & 1 & 1 & 0 & 0 & 1 \\
1 & 1 & 1 & 0 & 0 & 1 & 0 & 0 \\
0 & 0 & 1 & 0 & 0 & 1 & 1 & 1 \\
1 & 0 & 0 & 1 & 1 & 0 & 1 & 0
\end{array}\right] c_{c_{1}}^{c_{2}} \quad, \mathrm{x}=1,2, \ldots \mathrm{N} \text { and } \mathrm{y}=1,2, \ldots \ldots, \mathrm{M}
$$

Fig. 2 depicts another configuration of the $\mathrm{H}$ matrix where the Tanner graph contained check nodes sets and variable nodes that corroborate each matrix row and column, respectively.

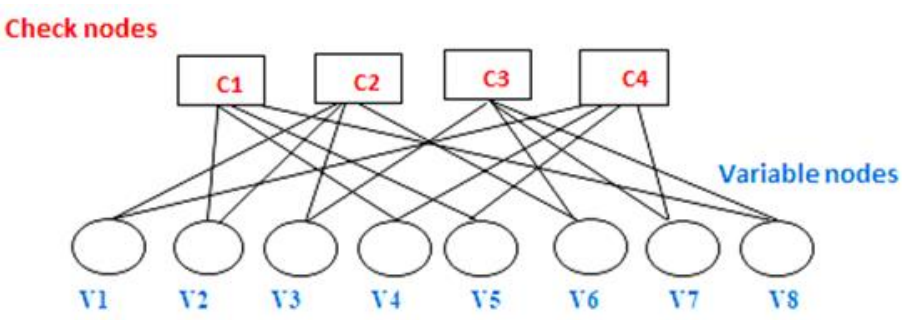

Fig. 2. A graphical representation of LDPC codes

\section{System Model}

The optimal parameters of LDPC code with DiPPM format have been investigated using slope detection and central detection techniques. However, it is vital to building an accurate system model to carry out a successful simulation. A system model describes the relationships between the inlet and outlet parameters. Fig. 3 represents a block diagram of the receiver system that signifies the FEC communication system, which is reliant on the LDPC error-control code.

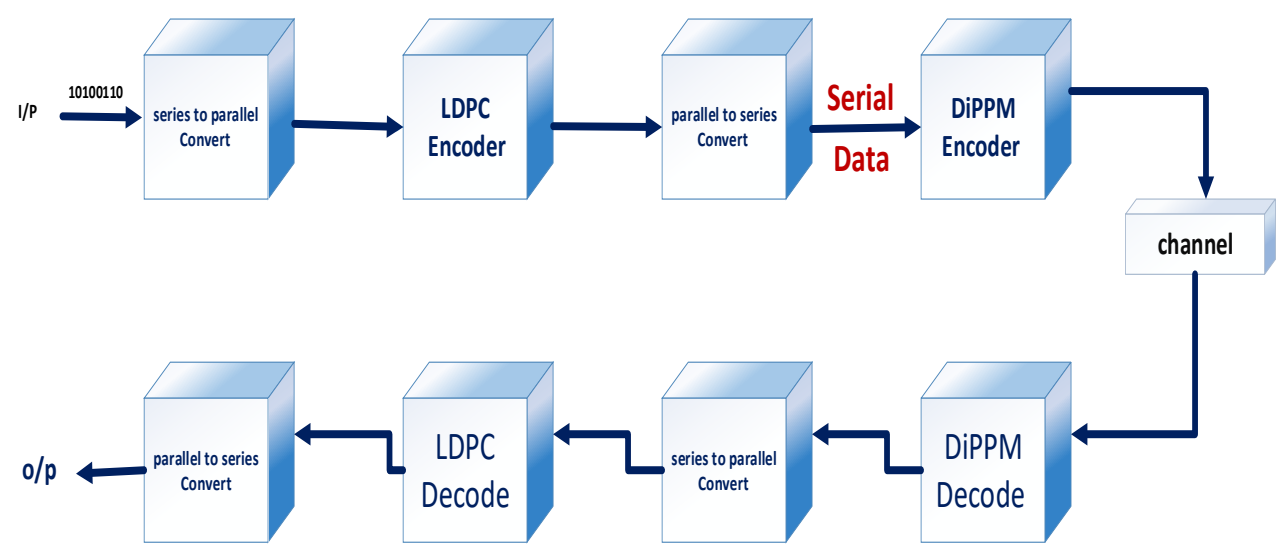

Fig. 3. System model.

This system characterizes by using a new device variable denotes as $v$ (Eq. 1) that signifies the division of the decision voltage to the peak signal voltage. Therefore, the system has capsulated both the peak voltage of the signal $\left(v_{p k}\right)$ and threshold crossing voltage $\left(v_{d}\right)$ as illustrated below $v=\frac{v_{d}}{v_{p k}}$ 
The effectiveness of transmission $(\rho)$ for both uncoded and coded DiPPM (when the LDPC code is used) can be calculated using Eqs. 2 and 3, respectively, based on the number of photons per pulse $(b)$. It is important to note that using an LDPC code would reduce the system's transmission efficacy based on the code rate $(r)$, as depicted in Eq. 3. Thus, it is important to reduce $b$ and utilize the optimal code rate in the deployment of an LDPC code to guarantee an accepted transmission efficacy [11].

$\rho=\frac{\ln 2}{b}$

$\rho=\frac{r \ln 2}{b}$

Eq. 4 is used to compute the bandwidth expansion for coded DiPPM

$$
B W E_{\text {coded DiPPM }}=\frac{n}{k} \times B W_{D i P P M}
$$

The simulation has considered a white-noise spectrum at the output of an optical receiver with restricted bandwidth as depicted in Fig. 3. Specifically, the pre-detection filter was used as a feasible matched filter due to having a receiver of a white-noise spectrum. The signal obtainable to the threshold detector was considered for transmission of dicode PPM via graded-index POF. Accordingly, the time variation of the device variable $\left(v_{o}(t)\right)$ is mathematically represented as

$$
v_{o}(t)=b \eta q R_{T} \frac{\omega_{c}}{2} \exp \left(\alpha^{2} \omega^{2}\right) \times \exp \left(-\omega_{c} t\right) \operatorname{erfc}\left[\alpha \omega_{c}-\left(\frac{t}{2 \alpha}\right)\right]
$$

$\eta$ is the detector's quantum efficiency, $q$ is the electronic charge, $R_{T}$ is the receiver's mid-band trans-impedance, and $\omega$ is the change of the Gaussian pulse obtained. $\alpha$ is the fibre bandwidth that can be estimated from Eq. 6

$\alpha=\frac{0.1874 T_{b}}{f_{n}}$

$T_{b}$ is the bit-time of PCM and $f_{n}$ is the fibre bandwidth normalised to the PCM data rate. $\left(n_{o}\right)$ signifies the noise performing on the signal as given in the context of Eq. 7

$$
\left\langle n_{o}^{2}\right\rangle=S_{o} \frac{\omega_{c}}{2} R_{T}^{2} \exp \left(\alpha^{2} \omega_{c}^{2}\right) \operatorname{erfc}\left(\alpha \omega_{c}\right)
$$

$S_{o}$ is the equivalent double-sided input-noise current spectral density of the preamplifier. The distribution of the channel log-likelihood ratio (LLR) is used to analyse LDPC codes. The channel LLR L is frequently measured by supposing the code words are all zeros. $(x=+1)$ was conveyed and therefore $\mathrm{L}$ is clearly expressed as

$\mathrm{L}=\log \frac{p(x=+1 \mid y)}{p(x=-1 \mid y)}$

LLRs have asymmetric Gaussian distribution for the BIAWGN( $\sigma)$ with mean $\mu=2 / \sigma 2$ and variance $4 / \sigma 2$. A detection error exists with negative values of LLR. This study uses $N$ samples of this distribution in each realization of the channel compared to a pure Gaussian LLR distribution. Thus, there is no consistency between pure Gaussian distribution and LLR distribution with a considerable diverge. However, the LLR distribution and the channel parameter remain constant. It is worth noting that a single parameter would not be able to completely describe this deviation.

The probability distribution of average bit error for the finite-length case is computed by considering the $\mathrm{N}$ samples from the symmetric Gaussian LLR distribution. In this regard, each sample orbit is incorrect within negative LLR. Accordingly, the likelihood of error of each sample (Fpobs) is mathematically defined on $\mathrm{x}$ in the following equation (Eq. 9). In this regard, the summation of all corresponding errors divided by $\mathrm{N}$ would signify the whole probability of errors.

$$
\mathrm{Fp}_{\mathrm{obs}}(\mathrm{x})=\left(\begin{array}{c}
N \\
N x
\end{array}\right) \mathrm{p}_{o}^{N x}\left(1-\mathrm{p}_{0}\right)^{\mathrm{N}-\mathrm{Nx}}
$$


$\mathrm{N} \mathrm{x}$ is an integer, and $0 \leq \mathrm{N} \mathrm{x} \leq \mathrm{N}$. However, The Fpobs is approximated defined by a Gaussian pdf $\mathrm{N}(\mathrm{p} 0, \mathrm{p} 0(1 \mathrm{p} 0) / \mathrm{N})$ with insignificant values for $\mathrm{x}$ outside $[0,1]$ for large $\mathrm{N}$.

\section{Results and Discussion}

The intension of this section is to focus on discussing the influence of the number of photons per pulse (b) in the DiPPM coded format using slope and central detection systems at different code speeds. The number of photons is measured using a $2 \mathrm{M}$ code word length, besides considering $M$ values between 3 to 7 . It is important to realize that $b$ increases as the LDPC code rate raises. This is due to the consistent correlation between the LDPC code rates and the number of data symbols. Also, $\mathrm{b}$ is proportionate to the normalised bandwidth when using the slope detection process. This is also due to the reliance on the slope detection approach to the form of established signal. Fig. 4 demonstrates the variation of $b$ for DiPPM system using LDPC against LDPC code rate at different normalised bandwidths. The minimum numbers of photons can be determined at different LDPC code rates and different normalised bandwidths. It is worth noting that the system kept the number of errors to an absolute minimum. More importantly, $\mathrm{b}$ is inversely proportionate to the number of normalised bandwidths as depicted in Fig. 4. Specifically, the lowest $\mathrm{b}$ is confirmed for the highest normalised bandwidth. Also, there is an exponential increase of $b$ against the increase of the LDPC code rate. In this regard, it is vital to perceive the importance of mitigating $b$ to accordingly generate the optimal code rate in the application of an LDPC code and to accelerate the highest performance of the device.

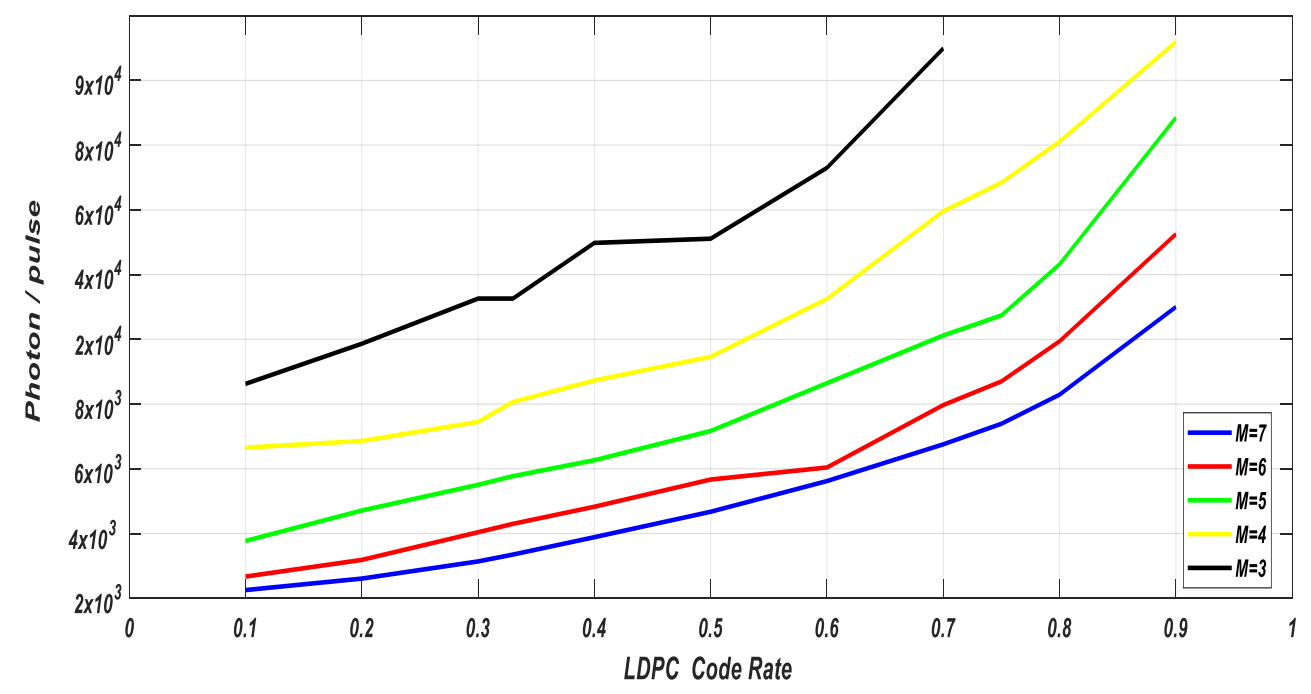

Fig. 4. Number of photons against LDPC code rate at different normalised bandwidths

Fig. 5 depicts the coded DiPPM system's transmission efficacy against LDPC code rates for several fibre normalised bandwidths. Fig. 5 confirms an optimum transmission efficacy at approximately 0.6 of LDPC code rate for the highest normalised bandwidths. However, the optimum transmission efficacy exists within higher LDPC code rates of approximately 0.7 for the lower normalised bandwidths. It is important to perceive the existence of an exponential relationship between the transmission efficacy and the code rate as the efficacy is reduced after gaining the optimal code rate. In other words, the uncoded symbol errors have increased even further due to an increase in code rate. Thus, the transmission of corrected symbols and the system's efficacy is reduced. In this regard, the redundancy, and the redundancy rate increase beyond the optimum code rate. However, higher fibre normalised bandwidths are successfully empowered the transmission efficacy.

Figs. 4 and 5 can also affirm the necessity of reducing $b$ with LDPC codes to applicably improve the transmission efficacy of the DiPPM. 


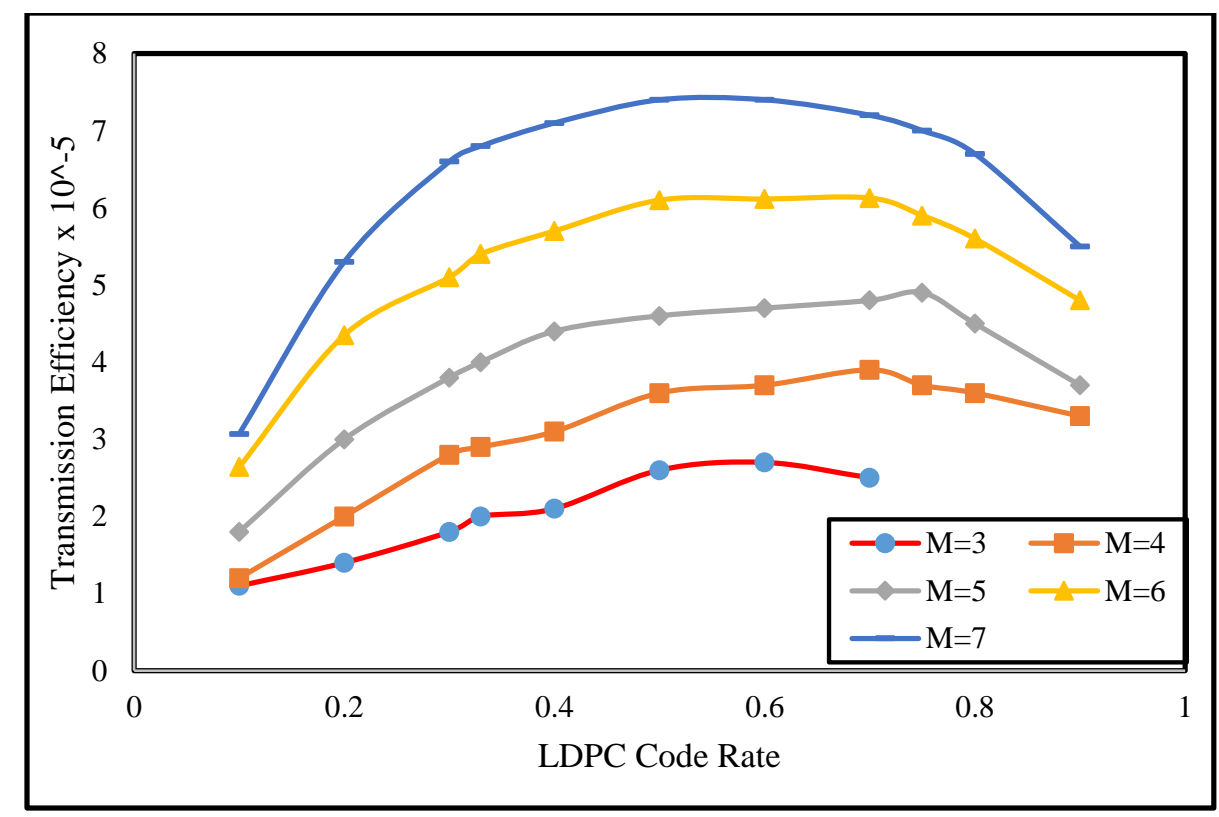

Fig. 5. The transmission efficacy of coded DiPPM system against LDPC code rates within different normalised bandwidths.

Fig. 6 demonstrates the variation of the number of photons per pulse for the coded PCM system against LDPC Code Rate for several normalised fibre bandwidths $\left(f_{n}\right)$. It can be stated that there is a direct positive relationship between the number of photons per pulse and the LDPC Code Rate for any selected normalised bandwidth. Specifically, the number of photons increases as the Code Rate increases for a particular bandwidth.

Fig. 7 clarifies the relationship between the transmission efficiency of PCM employing LDPC code system against the LDPC Code Rate for various fibre bandwidths $\left(f_{n}\right)$ normalised to the PCM data rate. It can be concluded that employing an optimum value of 0.7 of the Code rates would maximise the overall efficiency. The coded DiPPM using the LDPC code realizes the highest transmission efficiency for the low dispersive channel. This can be attributed to the bandwidth's extension which would consume more energy by adding the redundancy symbols. The calculation of transmission efficiency for the PCM using LDPC code system is carried out using the relationship of Sibley (2005) as follows

$\alpha=\frac{0.1874 T_{b}}{f_{n}}$

$T_{b}$ is the PCM bit-time. The noise acting on this signal is elucidated in the following equation

$\rho=\frac{r \ln 4}{b}$ 


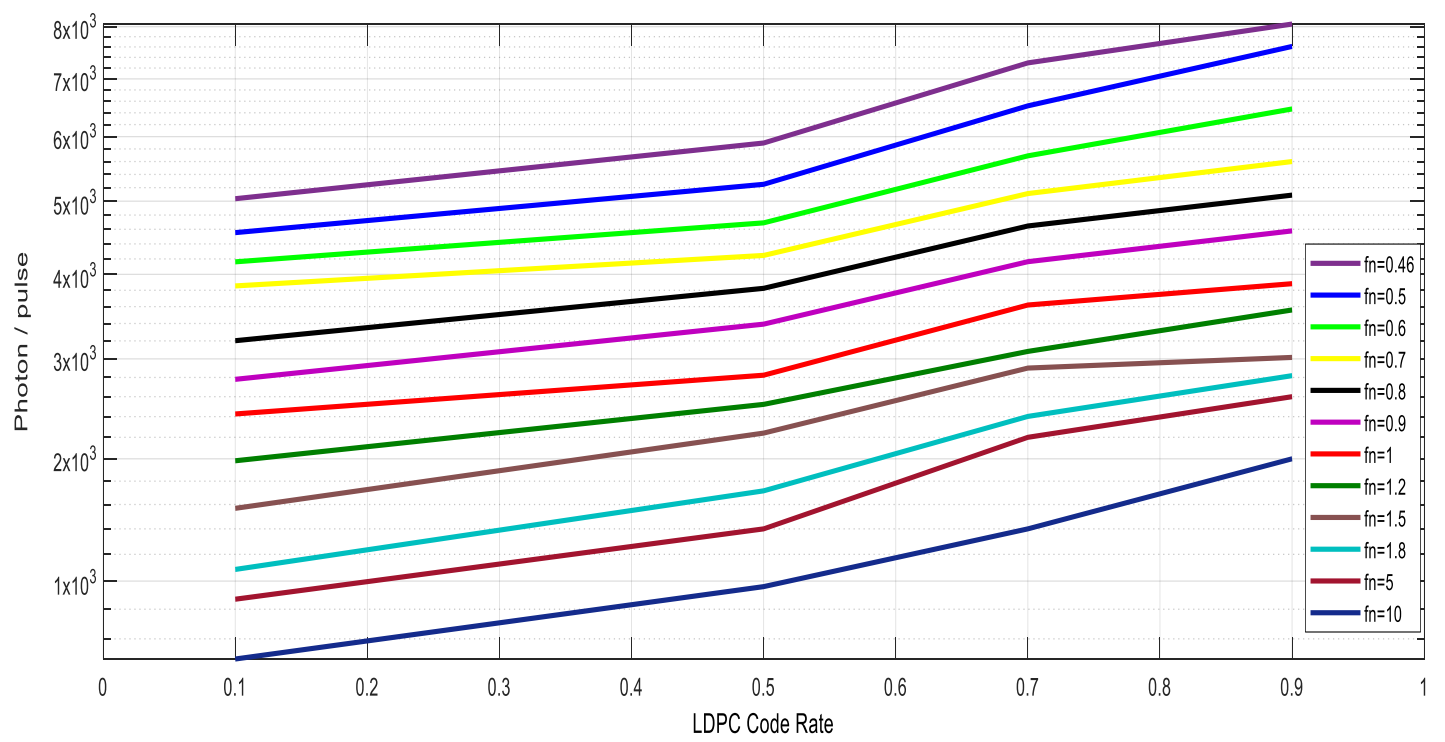

Fig. 6. Variation of photons' number against LDPC code rate at different normalised bandwidth.

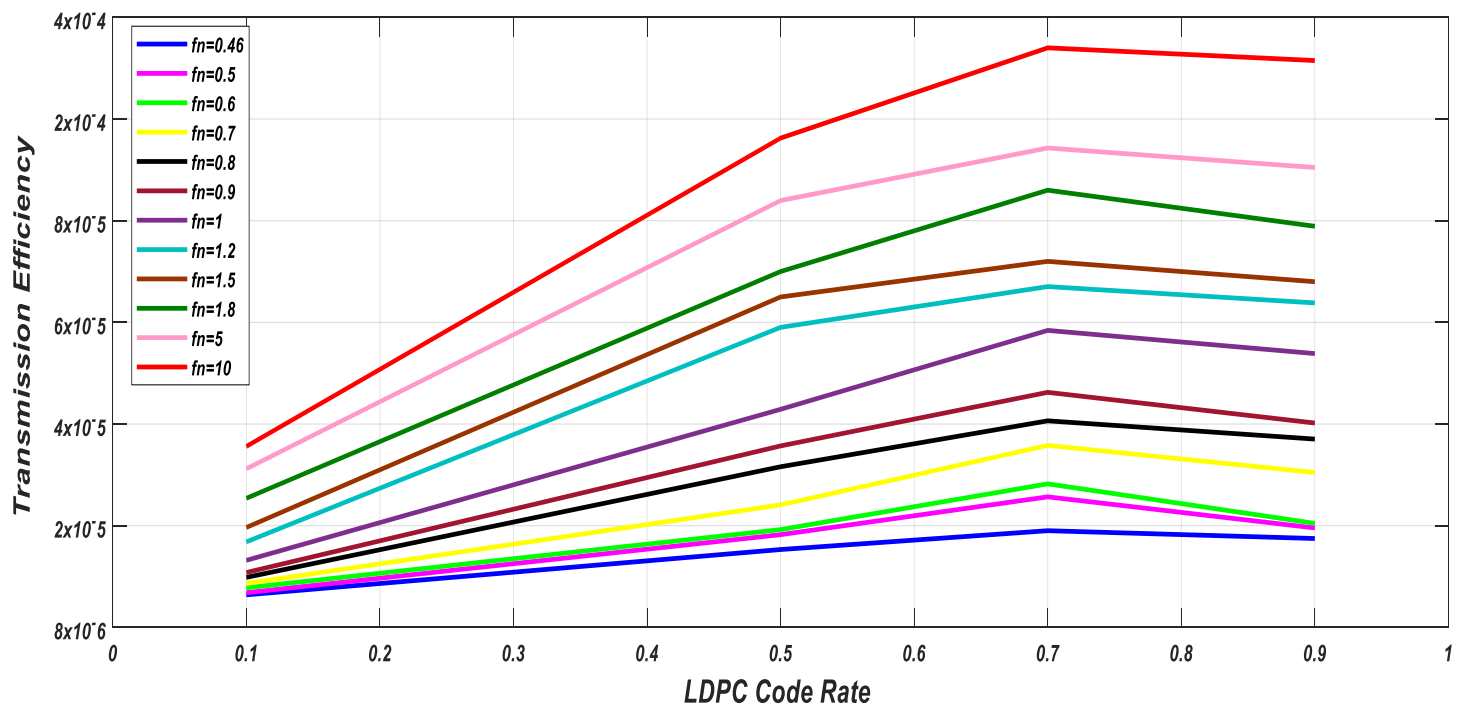

Fig. 7. Transmission efficiency versus LDPC code rate at different normalised bandwidth

\section{Conclusions}

LDPC codes with DiPPM were investigated in this paper for improving the transmission efficacy, bandwidth expansion, and several photons necessitated per pulse. The DiPPM coded system outperforms the uncoded system. To manage the main aim of this study, the obtained signal was detected using two improved techniques of the slope detection and central detection. It can be stated that reducing the number of photons with LDPC codes would significantly enhance the DiPPM's transmission efficacy. Furthermore, the machine efficiency can be improved by increasing the LDPC codeword. More importantly, the study delivered the optimum LDPC code rates for high and low fibre normalised bandwidths that entailed the maximum transmission efficacy.

\section{References}

[1] Al-Nedawe, B. M., Buhafa, A.M., Sibley, M.J., and Mather, P.J., 2013. Improving error performance of dicode pulse position modulation system using forward error correction codes. In 201321 st Telecommunications Forum Telfor (TELFOR) (pp. 331-334). IEEE.

[2] Charitopoulos, R. and Sibley, M.J., 2009. Experimental coder/decoder of dicode pulse position modulation. University of Huddersfield. 
[3] Ghassemlooy, Z., Hayes, A.R. and Wilson, B., 2003. Reducing the effects of intersymbol interference in diffuse DPIM optical wireless communications. IEE Proceedings-Optoelectronics, 150(5), pp.445-452. [4] Li, E., Declercq, D., and Gunnam, K., 2013. Trellis-based extended min-sum algorithm for non-binary LDPC cod es and its hardware structure. IEEE Transactions on Communications, 61(7), 2600-2611.

[5] Li, H., Liu, H., and Vafi, S., 2014. Bipolar chaotic pulse position modulation communication system based on cyclic LDPC. EURASIP Journal on Wireless Communications and Networking, 2014(1), 1-9.

[6] Reed, I.S. and Solomon, G., 1960. Polynomial codes over certain finite fields. Journal of the society for industrial and applied mathematics, 8(2), pp.300-304.

[7] Sibley, M.J., 2003a. Analysis of dicode pulse position modulation using a PINFET receiver and a slightly/highly dispersive optical channel. IEE Proceedings-Optoelectronics, 150(3), 205-209.

[8] Sibley, M.J., 2003b. Dicode pulse-position modulation: a novel coding scheme for optical-fibre communications. IEE Proceedings-Optoelectronics, 150(2), 125-131. [

9] Sibley, M., 2005. Performance analysis of a dicode PPM system, operating over plastic optical fibre, using maximum likelihood sequence detection. IEE Proceedings-Optoelectronics, 152(6), 337-343.

[10] Wang, H., Su, Y., Zhang, G., and Zhang, T. (2007). Performance analysis for LDPC-coded optical PPM communication system in weak turbulence. In Second International Conference on Space Information Technology (Vol. 6795, p. 67956V). International Society for Optics and Photonics.

[11] Yazdani, R., and Ardakani, M., 2007. An efficient analysis of finite-length LDPC codes. In 2007 IEEE International Conference on Communications (pp. 677-682). IEEE.

[12] Hussein, Y. M., Mutlag, A. H., \& Al-Nedawe, B. M. (2021, June). Comparisons of Soft Decision Decoding Algorithms Based LDPC Wireless Communication System. In IOP Conference Series: Materials Science and Engineering (Vol. 1105, No. 1, p. 012039). IOP Publishing. 